CREAT. MATH. INFORM.

Volume 25 (2016), No. 2,

Pages $177-182$
Online version at https : //creative-mathematics. cunbm . utcluj. ro/

Print Edition: ISSN 1584 - 286X; Online Edition: ISSN 1843 - 441X

DOI: https://doi.org/10.37193/CMI.2016.02.09

\title{
Linear relations on the coefficients of the linking polynomial
}

\author{
KOKO K. KAYIBI ${ }^{1}$, S. PirZAdA ${ }^{2}$ and AhmAd M. Alghamdi ${ }^{3}$
}

ABSTRACT. In 1972, Brylawski showed that the coefficients of the Tutte polynomial of a matroid are not independent, but they obey some linear relations. This result was extended to matroid perspectives by Vergnas in 1999. We extend this result further to all matroids pairs, and we conjecture that all the linear relations obeyed by the coefficients of the linking polynomial are linear combinations of the "basic" ones.

\section{INTRODUCTION}

Let $M$ be a matroid defined on a set $E$. The Tutte polynomial of $M$ is a 2-variable polynomial defined as

$$
T(M ; x, y)=\sum_{X \subseteq E}(x-1)^{r(E)-r(X)}(y-1)^{|X|-r(X)},
$$

where $r$ is the rank function of $M$. For an exposure into this topic, see $[2,3,4,5,17]$. The Tutte polynomial can be expanded as

$$
T(M ; x, y)=\sum_{i j} T_{i j} x^{i} y^{j}
$$

It is shown by Brylawski [1] that the coefficients $T_{i j}$ are not independent, but they obey linear relations given in the following theorem.

Theorem 1.1. [1] If $M$ is a matroid defined on a set $E$ such that $|E|>m$ for some integer $m$, and $T(M ; x, y)=\sum_{i, j} T_{i j} x^{i} y^{j}$ then the following identity holds among the coefficients $T_{i j}$ :

$$
I_{m}(M)=\sum_{i=0}^{m-1} \sum_{j=0}^{m-i-1}(-1)^{j}\left(\begin{array}{c}
m-i-1 \\
j
\end{array}\right) T_{i j}=0 .
$$

Furthermore, in the vector space $V$ of linear combinations of Tutte coefficients $\left\{T_{i j}\right\}$, the vectors $\left\{I_{1}, \ldots, I_{m}, \ldots\right\}$ form a basis for the subspace of all linear identities which hold for all but a finite number of Tutte polynomials.

Now, let $M \rightarrow N$ be a matroid perspective defined on a set $E$ with rank functions $r$ and $s$ for $M$ and $N$, respectively. Theorem 1.1 was extended to the perspective polynomial $t(M \rightarrow N ; x, y, z)$ by Vergnas [11] as follows.

Received: 23.01.2016. In revised form: 01.04.2016. Accepted: 06.05.2016

2010 Mathematics Subject Classification. 05C30, 68R10.

Key words and phrases. Matroid pairs, matroid perspectives, linking polynomial, Tutte polynomial, linear combination.

Corresponding author: S. Pirzada; pirzadasd@kashmiruniversity.ac.in 
Theorem 1.2. [11] Let $t_{i j k}$ denotes the coefficient of $x^{i} y^{j} z^{k}$ in the Tutte polynomial $t(M \rightarrow$ $N ; x, y, z)$ of a matroid perspective $M \rightarrow N$. For non negative integers $m, d$, let

$$
\begin{aligned}
f_{m} & =\sum_{0 \leq i+j \leq m, k \geq 0}(-1)^{i+k}\left(\begin{array}{c}
m-j+k \\
i+k
\end{array}\right) t_{i j k}, \\
f_{m, d} & =\sum_{0 \leq i+j \leq m, 0 \leq k \leq d}(-1)^{i+k}\left(\begin{array}{c}
m-j+k \\
i+k
\end{array}\right) t_{i j k} .
\end{aligned}
$$

If the number of elements of $M$ (or $N$ ) is at least $m+1$ then the coefficients in $t(M \rightarrow N)$ satisfy the relation $f_{m}=0$.

Conversely, $\left\{f_{m, d}\right\}$ for $m=0,1 \ldots$ constitutes a basis of the vector space of linear forms $f$ in finitely many variables $t_{i j k}$ with $i, j=0,1 \ldots$ and $0 \leq k \leq d$ such that $f=0$ is satisfied by the coefficients of Tutte polynomials of all but a finite number of matroid perspectives $M \rightarrow N$ with $r(E)-s(E)=d$.

We present an extension for any matroid pair. Indeed, for two matroids $M$ and $N$ defined on the set $E$ and having rank functions $r$ and $s$, respectively, the linking polynomial, denoted by $Q(M, N ; x, y, u, v)$, is defined as follows.

$$
Q(M, N ; x, y, u, v)=\sum_{X \subseteq E}(x-1)^{r(E)-r(X)}(y-1)^{|X|-r(X)}(u-1)^{s(E)-s(X)}(v-1)^{|X|-s(X)} .
$$

The linking polynomial, studied in $[6,7,8,18]$, is essentially equivalent to the 3 -variable polynomial defined and studied by Las Vergnas in a series of papers [11, 12, 13, 14, 15, 16]. It also partially contains the Tutte invariant of 2-polymatroids defined by Oxley and Whittle in $[9,10]$. Let the linking polynomial be expanded as

$$
Q(M, N ; x, y, u, v)=\sum_{i j k l} q_{i j k l} x^{i} y^{j} u^{k} v^{l} .
$$

We present the linear relations obeyed by the coefficients $q_{i j k l}$.

\section{MAIN RESULTS}

Theorem 2.3. Let $(M, N)$ be a matroid pair defined on a set $E$ with $|E|=n$. Let the rank functions be $r$ and $s$ for $M$ and $N$, respectively. For non negative integer $m$, let

$$
\Pi_{n, m}=\sum_{i=0}^{r(E)} \sum_{j=0}^{n} \sum_{k=0}^{s(E)} \sum_{l=0}^{n} \sum_{\tau=0}^{\tau_{1}} \sum_{\sigma=0}^{\sigma_{1}} q_{i j k l} 2^{\tau}(-1)^{n-m-l+\sigma}\left(\begin{array}{c}
i+j \\
\tau
\end{array}\right)\left(\begin{array}{c}
k+l \\
\sigma
\end{array}\right)\left(\begin{array}{c}
n-j \\
m+i+l-\tau-\sigma
\end{array}\right),
$$

where $\tau_{1}=\min \{m+i+l, i+j\}$ and $\sigma_{1}=\min \{m+i+l-\tau, k+l\}$.

Then, for all non negative integers $m$ such that $n-r(E)+s(E)>m$ the coefficients of $Q(M, N, x, y, u, v)$ obey the linear relations $\Pi_{n, m}=0$.

In order to prove this theorem, we need some preliminary lemmas. The next lemma is just a special case of Equation (3) of [18].

Lemma 2.1. Let $(M, N)$ be a matroid pair defined on the set $E$ with $|E|=n$ such that $r$ and $s$ are the rank functions of $M$ and $N$, respectively. Then for all real numbers $\alpha$ not equal to 0 or 1 the following holds:

$$
Q\left(M, N ; \frac{2 \alpha-1}{\alpha}, \frac{2 \alpha-1}{\alpha-1}, \alpha+1, \frac{\alpha+1}{\alpha}\right)=\alpha^{n+s(E)-r(E)}(\alpha-1)^{r(E)-n} .
$$


Let a function, denoted by $p(\alpha)$, be defined as

$$
p(\alpha)=\sum_{i=0}^{r(E)} \sum_{j=0}^{n} \sum_{k=0}^{s(E)} \sum_{l=0}^{n} q_{i j k l}(2 \alpha-1)^{i+j} \alpha^{-i-l}(\alpha-1)^{n-j}(\alpha+1)^{k+l} .
$$

Lemma 2.2. Let $(M, N)$ be a pair of matroids defined on the set $E$ with $|E|=n$. Let $m$ be an integer such that $m<n-r(E)+s(E)$. Then the coefficients of $Q(M, N ; x, y, u, v)$ obey a set of relations given by equating to zero the coefficients of $\alpha^{m}$ in the polynomial $p(\alpha)$.

Proof. In what follows, the expression $\sum_{i, j, k, l}$ stands for $\sum_{i=0}^{r(E)} \sum_{j=0}^{n} \sum_{k=0}^{s(E)} \sum_{l=0}^{n}$. By Lemma 2.1, we get equation (2.3). On the other hand it is routine to check, by rearranging the powers, that

$$
Q\left(M, N ; \frac{2 \alpha-1}{\alpha}, \frac{2 \alpha-1}{\alpha-1}, \alpha+1, \frac{\alpha+1}{\alpha}\right)=\sum_{i, j, k, l} q_{i j k l}(2 \alpha-1)^{i+j} \alpha^{-i-l}(\alpha-1)^{-j}(\alpha+1)^{k+l}
$$

Thus, using Equations (2.3) and (2.4) we get

$$
\sum_{i, j, k, l} q_{i j k l}(2 \alpha-1)^{i+j} \alpha^{-i-l}(\alpha-1)^{-j}(\alpha+1)^{k+l}=\alpha^{|E|+s(E)-r(E)}(\alpha-1)^{r(E)-n},
$$

which can be rewritten as

$$
\sum_{i j k l} q_{i j k l}(2 \alpha-1)^{i+j} \alpha^{-i-l}(\alpha-1)^{n-j}(\alpha+1)^{k+l}=\alpha^{n+s(E)-r(E)}(\alpha-1)^{r(E)} .
$$

But, for all matroids defined on a set $E$ with $|E|=n$ and rank function $r$, we have $n-r(E) \geq 0$. Thus the right hand side of equation (2.5) is a polynomial in $\alpha$, as $n-r(E)+$ $s(E)$ is always non negative. Therefore the left hand side of Equation (2.5), which is $p(\alpha)$, is also a polynomial in $\alpha$.

Now, let $m$ be an integer such that $n-r(E)+s(E)>m$. All the exponents of $\alpha$ in the polynomial in the right hand side of Equation (2.5) are greater than $m$. Thus the coefficient of $\alpha^{m}$ is zero in the right hand side of equation (2.5). This implies that the sum over all the coefficients of $\alpha^{m}$ must also be zero in the left hand side of Equation (2.5). This gives a relation obeyed by the coefficients of $q_{i j k l}$ in the left hand side.

The next lemma is a basic property of the binomial coefficients.

Lemma 2.3. Let $a_{1}, a_{2}, a_{3}, b_{1}, b_{2}, b_{3}, m_{1}, m_{2}, m_{3}, m$ be integers, an let $x$ be an indeterminate. Then, for all $m \leq m_{1}+m_{2}+m_{3}$, the coefficient of $x^{m}$ in the polynomial $\left(a_{1} x+b_{1}\right)^{m_{1}}\left(a_{2} x+\right.$ $\left.b_{2}\right)^{m_{2}}\left(a_{3} x+b_{3}\right)^{m_{3}}$ is given by

$$
\sum_{\tau=0}^{m} \sum_{\sigma=0}^{m-\tau}\left(\begin{array}{c}
m_{3} \\
\tau
\end{array}\right)\left(\begin{array}{c}
m_{2} \\
\sigma
\end{array}\right)\left(\begin{array}{c}
m_{1} \\
m-\tau-\sigma
\end{array}\right) a_{3}^{\tau} b_{3}^{m_{3}-\tau} a_{2}^{\sigma} b_{2}^{m_{2}-\sigma} a_{1}^{m-\tau-\sigma} b_{1}^{m_{1}-m+\sigma+\tau} .
$$

Proof of Theorem 2.3. First of all, we observe that the coefficient of $\alpha^{m}$ in the left hand side of Equation (2.5) is the coefficient of $\alpha^{m+i+l}$ in the polynomial

$$
\sum_{i, j, k, l} q_{i j k l}(2 \alpha-1)^{i+j}(\alpha-1)^{n-j}(\alpha+1)^{k+l} .
$$

By Lemma 2.2, one gets some relations obeyed by the coefficients of $Q(M, N)$ by equating to zero the coefficient of $\alpha^{m}$ in $p(\alpha)$ for $m<n-r(E)+s(E)$. Now using Lemma 2.3 
on equation (2.6), where we make the substitutions $a_{1}=1, a_{2}=1, a_{3}=2, b_{1}=-1, b_{2}=1$, $b_{3}=-1, m_{1}=n-j, m_{2}=k+l, m_{3}=i+j$, we get

$$
\sum_{i=0}^{r(E)} \sum_{j=0}^{n} \sum_{k=0}^{s(E)} \sum_{l=0}^{n} \sum_{\tau=0}^{m+i+l m+i+l-\tau} \sum_{\sigma=0} q_{i j k l} 2^{\tau}(-1)^{n-m-l+\sigma}\left(\begin{array}{c}
i+j \\
\tau
\end{array}\right)\left(\begin{array}{c}
k+l \\
\sigma
\end{array}\right)\left(\begin{array}{c}
n-j \\
m+i+l-\tau-\sigma
\end{array}\right)=0 .
$$

But, we know that $\left(\begin{array}{c}i+j \\ \tau\end{array}\right)=0$ for all $\tau>i+j$. Similarly, $\left(\begin{array}{c}k+l \\ \sigma\end{array}\right)=0$ for all $\sigma>k+l$. Thus we can limit the upper bound of the summations over $\tau$ to $\min \{m+i+l, i+j\}$ and over $\sigma$ to $\min \{m+i+l-\tau, k+l\}$ in order to eliminate the zero terms.

Examples. Consider the class of matroid pairs $(M, N)$ such that $|E|=1$ and $|E|-r(E)+$ $s(E) \geq 1$. This class contains the pairs $(C, C),(L, C),(L, L)$, where $C$ and $L$ denote a coloop and a loop, respectively. It is routine to check that

$$
\Pi_{1,0}=-q_{0000}-3 q_{1000}-q_{0100}-q_{0010}-2 q_{1010}+q_{0101}=0 .
$$

We hasten to say that the relation (2.7) holds for the coefficients of all $Q(M, N)$, where the pair $(M, N)$ satisfy the condition $|E|-r(E)+s(E) \geq 1$.

But, consider the matroid pair $(C, L)$. Here $|E|+s(E)-r(E)=0$. Thus it seems impossible to use Theorem 2.3 in this case. But we know that

$$
Q(C, L ; x, y, u, v)=Q(L, C ; u, v, x, y) .
$$

Thus, the linear relations obeyed by the coefficients of $Q(C, L)$ can be deduced from those of $Q(L, C)$. This is the object of the following result.

Corollary 2.1. Let $m$ be a nonnegative integer. Let $\mathcal{P}_{n, m}$ be the class of all the matroid pairs defined on $E$ with $|E|=n$ and whose $Q$-coefficients obey the linear relation $\Pi_{n, m}$. Let $\mathcal{P}_{n, m}^{s}$ be the class of pairs $(N, M)$ for all pairs $(M, N) \in \mathcal{P}_{n, m}$. Then $\mathcal{P}_{n, m}^{s}$ is the class of the matroid pairs whose $Q$-coefficients obey the relation $\Pi_{n, m}^{s}$ where $\Pi_{n, m}^{s}$ is obtained from $\Pi_{n, m}$ by interchanging the indices $i$ and $k, j$ and $l$ in $\Pi_{n, m}$.

Proof. If $(M, N) \in \mathcal{P}_{n, m}$ then, by the symmetry property of $Q$, we have

$$
Q(N, M ; x, y, u, v)=Q(M, N ; u, v, x, y) .
$$

Thus, if the coefficients of $Q(M, N ; x, y, u, v)$ obey the relation $\Pi_{n, m}$, the coefficients of $Q(N, M ; x, y, u, v)$ must obey the same relation, except that the index $i$ becomes $k, j$ becomes $l$ and vice versa.

Remark. One may have noticed that the expression $|E|-r(E)+s(E)$, which stems from Equation (2.3), is not symmetric in $r$ and $s$. But by the symmetry property of $Q$, we get that

$$
\begin{aligned}
Q\left(N, M ; \frac{2 \alpha-1}{\alpha}, \frac{2 \alpha-1}{\alpha-1}, \alpha+1, \frac{\alpha+1}{\alpha}\right) & =Q\left(M, N ; \alpha+1, \frac{\alpha+1}{\alpha}, \frac{2 \alpha-1}{\alpha}, \frac{2 \alpha-1}{\alpha-1}\right) \\
& =\alpha^{n+r(E)-s(E)}(\alpha-1)^{s(E)-n} \\
& =\sum_{i j k l} q_{i j k l}(2 \alpha-1)^{k+l} \alpha^{-j-k}(\alpha-1)^{-l}(\alpha+1)^{i+j} .
\end{aligned}
$$

Let us define a function $p^{\prime}(\alpha)$ as

$$
p^{\prime}(\alpha)=\sum_{i j k l} q_{i j k l}(2 \alpha-1)^{k+l} \alpha^{-j-k}(\alpha-1)^{n-l}(\alpha+1)^{i+j} .
$$


Using the above remark, we can have the equivalent form of Lemma 2.2 as follows.

Lemma 2.4. Let $(M, N)$ be a pair of matroids defined on the set $E$ with $|E|=n$. Let $m$ be an integer such that $m<n-s(E)+r(E)$. Then the coefficients of $Q(M, N ; x, y, u, v)$ obey a set of relations given by equating to zero the coefficients of $\alpha^{m}$ in the polynomial $p^{\prime}(\alpha)$.

With these preliminaries, and using the same argument as in the proof of Theorem 2.3, it is easy the prove the following.

Lemma 2.5. Let $(M, N)$ be a matroid pair defined on a set $E$ with $|E|=n$. Let the rank functions be $r$ and $s$ for $M$ and $N$, respectively. For non negative integer $m$, let

$$
\Pi_{n, m}^{\prime}=\sum_{i=0}^{r(E)} \sum_{j=0}^{n} \sum_{k=0}^{s(E)} \sum_{l=0}^{n} \sum_{\tau=0}^{\tau_{1}} \sum_{\sigma=0}^{\sigma_{1}} q_{i j k l} 2^{\tau}(-1)^{n-m-j+\sigma}\left(\begin{array}{c}
i+j \\
\sigma
\end{array}\right)\left(\begin{array}{c}
k+l \\
\tau
\end{array}\right)\left(\begin{array}{c}
n-l \\
m+k+j-\tau-\sigma
\end{array}\right),
$$

where $\tau_{1}=\min \{m+k+j, k+l\}$ and $\sigma_{1}=\min \{m+k+j-\tau, i+j\}$. Then, for all non negative integers $m$ such that $n-s(E)+r(E)>m$ the coefficients of $Q(M, N, x, y, u, v)$ obey the linear relations $\Pi_{n, m}^{\prime}=0$.

But, it is obvious that Equation (2.9) can be obtained from Equation (2.2) by just interchanging $r$ and $s, i$ and $k, j$ and $l$. Thus, for fixed $n$ and $m$, we have

$$
\Pi_{n, m}^{\prime}=\Pi_{n, m}^{s} .
$$

Similar to Corollary 2.1 we can use the duality property of $Q$ to get more relations between the coefficients.

Corollary 2.2. Let $m$ be a nonnegative integer. Let $\mathcal{P}_{n, m}$ be the class of all the matroid pairs defined on $E$ with $|E|=n$ and whose $Q$-coefficients obey the linear relation $\Pi_{n, m}$. Let $\mathcal{P}_{n, m}^{*}$ be the class of pairs $\left(M^{*}, N^{*}\right)$ for all matroid pairs $(M, N) \in \mathcal{P}_{n, m}$. Then $\mathcal{P}_{n, m}^{*}$ is the class of all the matroid pairs whose Q-coefficients obey the relation $\Pi_{n, m}^{*}$ where $\Pi_{n, m}^{*}$ is obtained from $\Pi_{n, m}$ by interchanging the indices $i$ and $j, k$ and $l$ in $\Pi_{n, m}$.

Proof. If $(M, N) \in \mathcal{P}_{n, m}$ then the duality property of $Q$ gives that

$$
Q\left(M^{*} N^{*} ; x, y, u, v\right)=Q(M, N ; y, x, v, u) .
$$

Thus, if the coefficients of $Q(M, N ; x, y, u, v)$ obey the relation $\Pi_{n, m}$, the coefficients of $Q\left(M^{*}, N^{*} ;, x, y, u, v\right)$ must obey the same relation, except that the index $i$ becomes $j, k$ becomes $l$ and vice versa.

Using Corollaries 2.1 and 2.2, we get

$$
\begin{aligned}
& \Pi_{1,0}^{s}=-q_{0000}-3 q_{0010}-q_{1000}-q_{0001}-2 q_{1010}+q_{0101}=0 \\
& \Pi_{1,0}^{*}=-q_{0000}-3 q_{0100}-q_{1000}-q_{0001}-2 q_{0101}+q_{1010}=0 .
\end{aligned}
$$

We suspect that we may have some kind of reduced form of the "basis" theorem of Brylawski and Las Vergnas as follows:

Conjecture Let $\mathcal{P}_{n}$ be the class of matroid pairs defined on $E$ with $|E|=n$. Then any linear identity which holds for all $Q$-polynomials in $\mathcal{P}_{n}$ is a linear combination of the relations

$$
\left\{\Pi_{n, 0}, \ldots, \Pi_{n, n-r(E)+s(E)-1}, \Pi_{n, 0}^{s}, \ldots, \Pi_{n, n-r(E)+s(E)-1}^{s}, \Pi_{n, 0}^{*}, \ldots, \Pi_{n, n-r(E)+s(E)-1}^{*}\right\} .
$$




\section{REFERENCES}

[1] Brylawski, T. H., A decomposition for combinatorial geometries, Trans. Amer. Math. Soc., 171 (1972), 1-44

[2] Brylawski, T. H., The Tutte polynomial, part 1: General theory, In A. Barlotti (ed). Matroid Theory and Its Applications, (C.I.M.E. 1980), Liguori, Naples, (1982), 125-275

[3] Brylawski, T. H., Oxley J., The Tutte polynomial and its applications, Matroid Application, N. L. White (Ed), Cambridge University Press, Cambridge, U. K, (1992), 123-225

[4] Crapo, H. H., The Tutte polynomial, Aeq. Math., 3 (1969, 211-229

[5] Fortuin, C. M. and Kasteleyn P. W., On the random-cluster model 1, Physica, 57 (1972) 536-564.

[6] Kayibi, K. K., A decomposition theorem for the linking polynomial, Disc. Math., 308 (2008) 583-597

[7] Kayibi, K. K., On the coefficients of the linking polynomial, Adv. Dev. Stat. Sci., 3 (2013), 45-56

[8] Kayibi, K. K. and Pirzada, S., On the activities of p-basis of matroid perspectives, Disc. Math., 339 (2016) No. 6 , 1629-1639

[9] Oxley, J. G. and Whittle, G., A characterization of Tutte invariants of 2-polymatroids, J. Combin. Theory, Ser., B, 59 (1993), 210-244

[10] Oxley, J. G., Whittle, G., Tutte invariants for 2-polymatroids, Contemporary Mathematics, Graph Structure Theory, (eds. N. Robertson and P. Seymour), 147 (1995), 9-20

[11] Vergnas, M. L., The Tutte polynomial of a morphism of matroids I. set-pointed matroids and matroid perspectives, Ann. Inst. Fourier, Grenoble, 49 (1999), No. 3, 973-1015

[12] Vergnas, M. L., On The Tutte polynomial of a morphism of matroids, Ann. Disc. Math., 8 (1980), 7-20

[13] Vergnas, M. L., The Tutte polynomial of a morphism of matroids, III. Vectorial matroids, Adv. App. Math., 32 (2004), No. 1, 198-211

[14] Vergnas, M. L., The Tutte polynomial of a morphism of matroids, 4. Computational complexity, Portugalia. Math., 64 (2007), 303-309

[15] Vergnas, M. L., The Tutte polynomial of a morphism of matroids, 5. Derivatives as generating functions of Tutte activities, Eur. J. Comb., 34 (2013), No. 8, 1390-1405

[16] Vergnas, M. L., The Tutte polynomial of a morphism of matroids, 6 . A multi-faceted counting formula for hyperplane regions and acyclic orientations, ArXiv: 1205.5424, (2012)

[17] Welsh, D. J. A., The Tutte polynomial, Random Struct. Alg., 15 (1999), 210-228

[18] Welsh, D. J. A., Kayibi, K. K., A linking polynomial of two matroids, Adv. Appl. Math., 32 (2004), 391-419

${ }^{1}$ Department of MATHEMATics AND Physics

UNIVERSITY OF HULL

Cottingham RD, Hull, YoRKshiRe HU6 7RX, United Kingdom

Email address: kokokayibi@gmail.com

${ }^{2}$ Department of MATHEMATICS

UNIVERSITY OF KASHMIR

HAZRATBAL, SRINAGAR, KASHMIR, INDIA

Email address: pirzadasdekashmiruniversity.ac.in

${ }^{3}$ DePartment of MATHEMATICAL SCIENCES

UMM ALQURA UNIVERSITY

MAKKAH, SAUDI ARABIA

Email address: amghamdi@uqu.edu.sa 